\title{
Temporal properties of rainfall events in Calabria (southern Italy)
}

\author{
O. G. Terranova and P. Iaquinta \\ National Research Council - Research Institute for Geo-Hydrologic Protection, Rende (Cosenza), Italy \\ Received: 17 June 2010 - Revised: 23 November 2010 - Accepted: 25 January 2011 - Published: 9 March 2011
}

\begin{abstract}
Temporal properties of 152575 rainfall events, recorded at time steps of $5 \mathrm{~min}$, having different durations and occurring between 1989 and 2008 at 155 localities of Calabria (Italy), have been analysed in this paper. Samples from 45533 storms have been selected to classify rainfall events as "significant" with regard to their contribution to soil erosion, flooding and/or other geo-hydrological processes. The samples are representative of a wide variety of situations in terms of duration, total rainfall, intensity, etc.

The use of standardized rainfall profiles (SRP) is proposed to describe the within-storm temporal pattern. The main attraction of this method lies in the fact that it is based on actual data of regional precipitation. Its weak point is that large samples of data are required to obtain regional profiles. The research necessities for improving the use of Huff curves for storm disaggregation and its the potential use are summarized in this paper on the basis of the specific literature.

A new criterion - based on the comparison of the areas $A_{1}, A_{2}, A_{3}$ and $A_{4}$ that underlie the four $25 \%$ of durations of a given SRP, and the corresponding four values of the "uniform" SRP (USRP), is suggested here with the aim of improving the use of the information content of SRP. Some interesting results concerning the sample frequency and the characterization of parameters for hydrological applications are commented on.

The study conducted so far has produced important, albeit preliminary, results for different contexts of Calabria concerning the use of SRP among the methods for constructing design storm hyetographs.
\end{abstract}

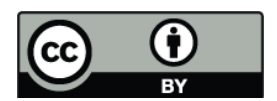

Correspondence to: O. G. Terranova (terranova@irpi.cnr.it)

\section{Introduction}

Many hydrological models require the rainfall pattern in the spatial-temporal domain as an input. In fact, quantities of more direct interest, such as the flow of a watercourse or the groundwater level or the rate of erosion of a basin, are frequently not directly available.

Moreover, rainfall observations are mainly available for intervals that are coarse in both space and time, while such information should be made known with adequate resolution. The quality of the input rainfall is crucial with relation to the model output.

In addition, the physical processes that determine rainfall are very complex. Models often try to reproduce the spatial and temporal distribution of available observations, but calibration is often unsatisfactory because of the high variability of rainfall intensity with time, as observed for several rain events (Kottegoda and Kassim, 1991).

The method of probabilistic representation introduced by Huff (1967) expresses, through the standardized rainfall profiles (SRP curves, or normalized mass curves, or Huff's curves), the nature of the randomness and the high variability of rainfall in time. The SRP can be seen as a random variable whose bidimensional function shows a relationship of the type:

$F_{D}(\pi, \tau)=P(\Pi \leq \pi, \Theta \leq \tau)$

in which $\pi=y_{t} / P_{\mathrm{ev}}$ and $\tau=t / D_{\mathrm{ev}}$ are the nondimensional depth and duration.

The distribution function $F_{D}$ can be seen as the occurrence probability of a SRP curve (Huff, 1967; Vukmirovich and Despotovic, 1983; Colosimo and Copertino, 1984; Colosimo et al., 1996). The availability of a statistically significant number, $N$, of SRP curves permits information to be derived in probabilistic terms.

Published by Copernicus Publications on behalf of the European Geosciences Union. 
At the generic dimensionless time $\tau_{i}$, the corresponding $N$ dimensionless rainfall, $\pi_{i, j}$, can be identified and statistically characterized; by repeating the analysis for different $\tau_{i}$, equal quantile curves can be obtained.

The analysis of the SRP can be performed to disaggregate the precipitation totals or to derive other types of information. To this purpose, the rainfall events can be classified according to various criteria: duration, total rainfall, maximum intensity in a fixed time or average intensity, geographical area of occurrence, etc. Moreover, different criteria are often applied in different environmental contexts, or in studies aimed at different purposes.

In the present paper, a total number of 152575 storms of different durations, occurred in different seasons between 1989 and 2008 and were recorded at time steps of $5 \mathrm{~min}$ at 155 rain gauges in Calabria and analyzed. The sample is representative of a wide variety of situations in terms of duration, total rainfall, intensity, etc. According to Wischmeier and Smith (1978), samples of 45533 storms were selected to classify rainfall events as "significant" with regard to their contribution to soil erosion, flooding and/or other geo-hydrological processes. Standardized rainfall profiles (SRP) were applied to characterize the selected rainfall events and obtain the design storms. A new criterion, based on the comparison between the areas $A_{1}, A_{2}, A_{3}$ and, $A_{4}$ (underlying the four $25 \%$ of duration of the SRP) and the corresponding four values of the uniform SRP (USRP), was defined to improve the use of the information content of SRP. Results concerning the sample frequency and the characterization of parameters for hydrological applications are discussed in the following.

\section{Data and methods}

\subsection{Main characteristics of rainfalls in Calabria}

In Calabria, average yearly rainfalls vary between 1000 and $2000 \mathrm{~mm} \mathrm{y}^{-1}$ in mountainous and internal areas, and between 600 and $900 \mathrm{~mm} \mathrm{y}^{-1}$ in coastal areas, with a mean regional value of about $1150 \mathrm{~mm} \mathrm{y}^{-1}$. As confirmed in a recent review of the general frame of storm conditions in Calabria, heavy rains are by far more frequent on the Jonian side of the region (Terranova, 2004). Over $70 \%$ of the yearly precipitation occurs from October to March, with negligible monthly values from June to September. Orography strongly influences the precipitation regime (Bellecci et al., 2002), due to fronts transversally approaching the Calabrian peninsula, and convective cells climbing up the steep sea-side slopes of the mountain chains.

\subsection{The data-set and Huff's analysis of SRP}

A 5-min rainfall database (ARPACal-Regione Calabria) has been available in Calabria since 1989; it is related to 155

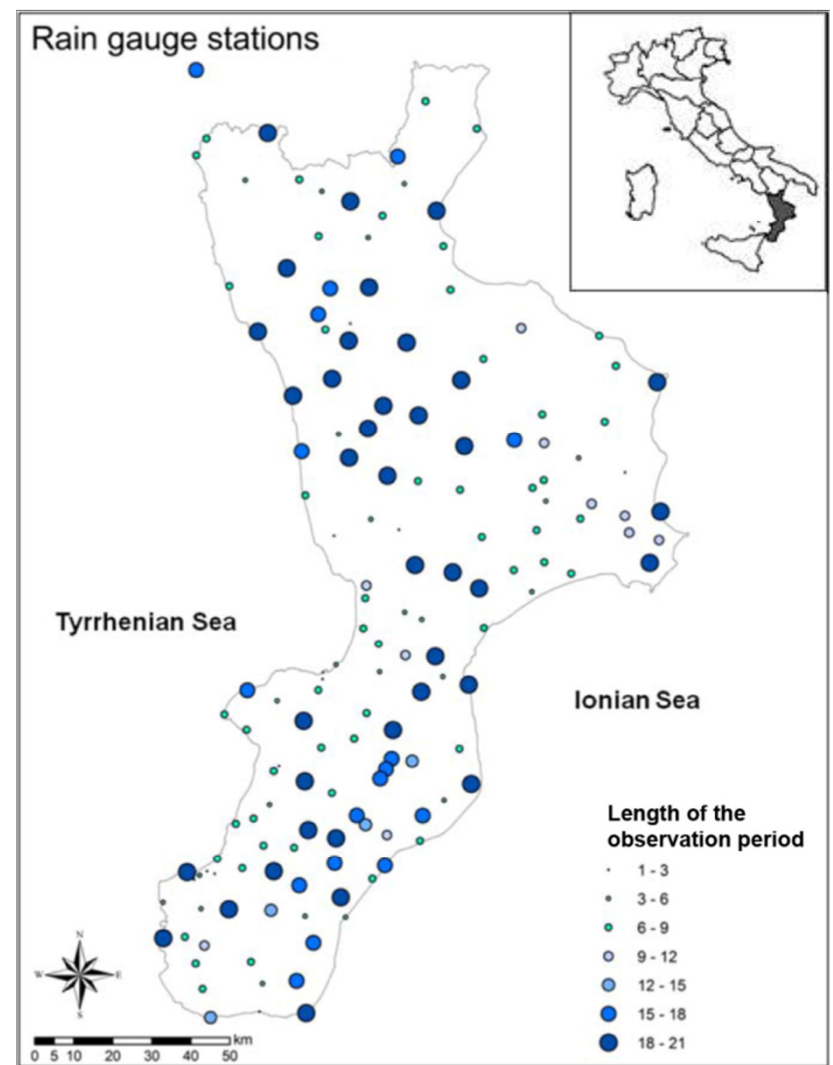

Fig. 1. Location of rain gauge stations and lengths of the observation period.

rain gauges consisting of an average of 12 years of observation, often affected by discontinuities (Fig. 1). A set of 152575 rainfall events on 155 stations is available, whereby each rainy period preceded and followed by least $6 \mathrm{~h}$ of dry weather is considered an "event". Such events were divided into 45533 erosive (ErREv: Erosive Rainfall Events) and 107042 non-erosive events (Wischmeier and Smith, 1978). In the following, attention is given exclusively to the study of the ErREv sub-set. These events are characterized by: (i) $P_{\mathrm{ev}}=23.5 \mathrm{~mm}$ as an average; (ii) 18033 events are related to $6 \mathrm{~mm} \leq P_{\mathrm{ev}}<12.7 \mathrm{~mm}$ but exceeding $6.35 \mathrm{~mm}$ in half-hour; (iii) 27501 events are related to $P_{\mathrm{ev}} \geq 12.7 \mathrm{~mm}$; (iv) $D_{\text {ev }}$ from $10 \mathrm{~min}$ to almost 9 days; (v) average $D_{\text {ev }}$ of $\sim 15 \mathrm{~h}$; (vi) maximum intensity in $30 \mathrm{~min}, I_{30}$, from negligible to $\sim 154.8 \mathrm{~mm} \mathrm{~h}^{-1}$; (vii) $I_{30}=11.6 \mathrm{~mm} \mathrm{~h}^{-1}$ in average.

As far as concerns the possibility of classifying the ErREv events to highlight their special features, a first distinction is given in Table 1 and a preliminary analysis of SRP is given in Table 2.

\subsection{A new criterion for classifying SRP}

In addition to the peak position in quartiles (Huff, 1967) and on the horizontal axis (NERC, 1975; Colosimo et al., 1996), 
Table 1. Adopted criteria of classification of the 45533 ErREv.

\begin{tabular}{lllllll}
\hline Quartiles & First & second & third & fourth & & \\
$D_{\mathrm{ev}}(\mathrm{h})$ & $<1$ & $\geq 1$ and $<3$ & $\geq 3$ and $<6$ & $\geq 6$ and $<12$ & $\geq 12$ and $<24$ & $\geq 24$ \\
$P_{\mathrm{ev}}(\mathrm{mm})$ & $<20$ & $\geq 20$ and $<30$ & $\geq 30$ and $<40 \quad \geq 40$ and $<60 \quad \geq 60$ and $<100$ & $\geq 100$ \\
$I_{30}\left(\mathrm{~mm} \mathrm{~h}^{-1}\right)$ & $<5$ & $\geq 5$ and $<7$ & $\geq 7$ and $<10$ & $\geq 10$ and $<14$ & $\geq 14$ and $<20$ & $\geq 20$ and $<30 \quad \geq 30$ \\
Period & Nov-Dec-Jan-Feb & May-Jun-Jul-Aug & & & & \\
\hline
\end{tabular}

Table 2. Characterization of SRP in relation to the criteria used to classify the 45533 ErREv.

\begin{tabular}{|c|c|}
\hline Observations & \\
\hline Quartiles & $\begin{array}{l}\text {-the largest number (15938) of SRP is for the first quartile } \\
\text {-the minimum number (8867) of SRP is for the fourth quartile } \\
\text {-2nd and 3rd quartiles show a smaller variability in the structure of rain compared to 1st and 4th quartile }\end{array}$ \\
\hline$D_{\mathrm{ev}}(\mathrm{h})$ & $\begin{array}{l}\text { for increasing values of } D_{\mathrm{ev}} \text {, the variability of the structure of rain increases for } D_{\mathrm{ev}} \leq 6 \mathrm{~h} \text {, then gradually } \\
\text { decreases for } D_{\mathrm{ev}}>6 \mathrm{~h} \text {; }\end{array}$ \\
\hline$P_{\mathrm{ev}}(\mathrm{mm})$ & $\begin{array}{l}\text { a progressive decrease in the variability of the structure of rain is observed when } P_{\mathrm{ev}} \text { increases from } \\
P_{\mathrm{ev}}<20 \mathrm{~mm} \text { to } P_{\mathrm{ev}}>100 \mathrm{~mm}\end{array}$ \\
\hline$I_{30}\left(\mathrm{~mm} \mathrm{~h}^{-1}\right)$ & $\begin{array}{l}\text { if } I_{30} \text { increases, the variability of the SRP structure is also increased with minimum value for } I_{30}<5 \mathrm{~mm} \mathrm{~h}^{-1} \\
\text { and maximum value for } I_{30}>30 \mathrm{~mm} \mathrm{~h}^{-1}\end{array}$ \\
\hline Period & for the dry season, the variability of the structure of rain is higher than for the wet season \\
\hline
\end{tabular}

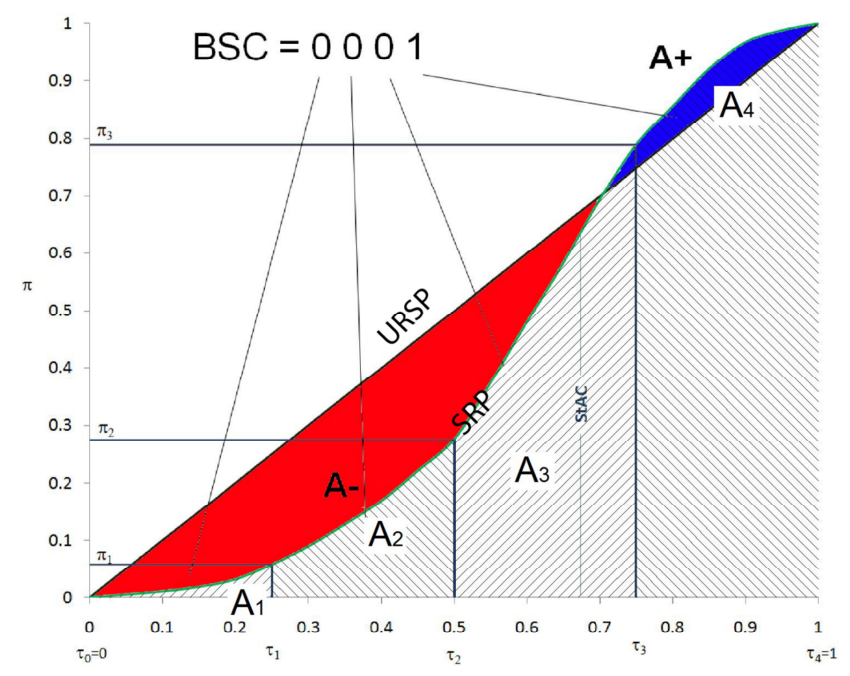

Fig. 2. Elements of a Standardized Rainfall Profile (SRP). USRP $=$ Uniform SRP; StAC $=$ Storm Advancement Coefficient, i.e. time of occurrence of the maximum rainfall intensity; total area under the SRP below the USRP, $A^{-}$(in red); total areas under the SRP above the USRP $A^{+}$(in blue); BSC=Binary Shape Code based on the comparison between the areas $A_{1}, A_{2}, A_{3}, A_{4}$ - underlying the four $25 \%$ of duration of the SRP - with the corresponding four values of the USRP. a different criterion was adopted to better identify the shape of the profile (Fig. 2) based on the comparison between the areas $A_{1}, A_{2}, A_{3}, A_{4}$ - underlying the four $25 \%$ of durations of the SRP - with the corresponding four values of the uniform SRP (USRP). More precisely, a 4-digit binary code was determined as follows:

i. Time values $\tau_{0}=0, \tau_{1}=0.25, \tau_{2}=0.50, \tau_{3}=0.75$, $\tau_{4}=1,\left(\tau_{k}\right.$, with $\left.k=0,1, \ldots, 4\right)$ are fixed on the horizontal axis;

ii. The corresponding USRP areas, $A_{k}^{*}=\left(1 / 2 \tau_{k}^{2}\right)-A_{k-1}^{*}$, with $A_{0}^{*}=0$, are calculated;

iii. SRP areas $A_{k}=\sum_{j=\tau_{k-1}}^{\tau_{k}} \frac{y_{j-1}+y_{j}}{2}\left(\tau_{k}-\tau_{k-1}\right)$, where $y_{j}$ represents the dimensionless height of accumulated rain corresponding to the abscissa $\tau_{k}$, are calculated;

iv. The binary code is built on the base of: $S_{k}=1$ if $A_{k}>$ $A_{k}^{*}$, else $S_{k}=0$;

v. Ultimately, the binary code $\mathrm{BSC}=S_{1} S_{2} S_{3} S_{4}$ is obtained.

The BSC code summarizes information of more immediate use than that provided by the: (i) distinction in quartiles (Huff, 1967); (ii) calculation of the "crossing properties" according to Kottegoda and Kassim (1991); (iii) recognition of the six "rainfall temporal patterns" proposed by Chukwuma 
Table 3. Correspondence between the proposed classification criteria and those of Kottegoda and Kassim (1991), Colosimo et al. (1996), Chuckwuma and Schwab (1983).

\begin{tabular}{|c|c|c|c|}
\hline BSC & $\begin{array}{l}\text { Type according to } \\
\text { Kottegoda and Kassim }\end{array}$ & $\begin{array}{l}\text { Code according to } \\
\text { Colosimo et al. (1996) }\end{array}$ & $\begin{array}{l}\text { Code according to } \\
\text { Chuckwuma and Schwab }\end{array}$ \\
\hline 0000 (lagged SRP) & $1 \mathrm{a}$ & $\mathrm{C}$ & $\mathrm{F}$ (or $\mathrm{E}$, or $\mathrm{D})$ \\
\hline 1111 (advanced SRP) & $1 b$ & B & $\mathrm{A}($ or $\mathrm{B}$, or $\mathrm{C})$ \\
\hline $0001,0011,0111$ & $2 \mathrm{a}$ & $\mathrm{E}$ & D (0001); C (0111) \\
\hline $1000,1100,1110$ & $2 b$ & $\mathrm{D}$ & B (1110); E (1000) \\
\hline $0100,0110,0010$ & $3 \mathrm{a}$ & & \\
\hline $1011,1001,1101$ & $3 b$ & & \\
\hline 0101 & $4 a$ & & \\
\hline 1010 & $4 \mathrm{~b}$ & & \\
\hline
\end{tabular}

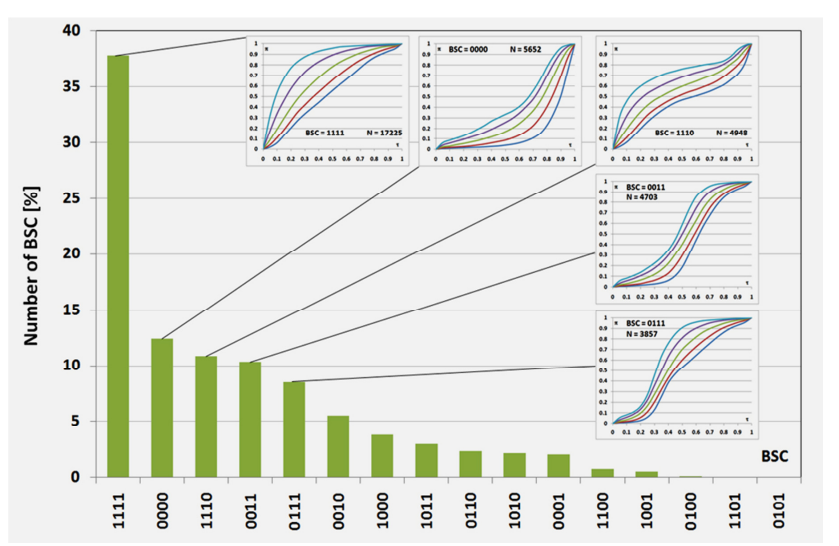

Fig. 3. Percentage of BSC relative to ErREv in Calabria and SRP relevant to the 5 most numerous BSC.

and Schwab (1983) or (iv) the distinction of the four storm structures proposed by Colosimo et al. (1996). In particular, the correspondences listed in Table 3 are valid.

Also, the total areas under the SRP below, $A^{-}$(Fig. 2, in red), and above, $A^{+}$(Fig. 2, in blue), the USRP were assessed in the present study (Kottegoda and Kassim, 1991). The classification into quartiles allows us to indicate the approximate location of the peak but does not provide information about the presence of secondary peaks or the time when the most important rains occur. For example, with regard to this last aspect, a BSC 0100 indicates that the $A^{+}$value can therefore be attributed primarily to the $\tau_{1}-\tau_{2}$ period and that $A^{-}$can be attributed to the remaining three periods. Other considerations, such as the rainfall intensity in the four intervals $\tau_{j-1}, \tau_{j}$, can be deduced from the comparison between SRP and USRP in relation to the values of $A^{+}, A^{-}$and BSC. More information on this aspect is provided by the Storm Advancement Coefficient (StAC) introduced by NERC (1975), i.e. the ratio between the time experiencing the maximum rain intensity and $D_{\text {ev }}$.

\section{Application of the new classification criterion of SRP to the calabrian database}

Regarding the classification of SRP, we can observe that: (i) BSC 1100, 1001, 0100, 1101, 0101 completed a total of less than $1.5 \%$ of all the observed SRP; (ii) the percentage rises to less than $8 \%$ of all the observed SRP when adding BSC 0110 , 1010, 0001; (iii) only 8 of 16 types of BSC occur with frequencies higher than $2.5 \%$; (iv) approximately $80 \%$ of SRP falls in BSC 1111, 0000, 1110, 0011, 0111. From Fig. 3, it can be seen that a higher frequency $(\sim 38 \%)$ competes to BSC 1111, and a virtually zero frequency to BSC 0101; the other four BSC $(0000,1110,0011,0111)$ have frequencies from 12.4 to $8.4 \%$. The $11 \mathrm{BSC}$ characterized by a frequency greater than $1 \%$ are analysed mainly in the following.

In some cases, the BSC can be related to specific weather conditions. In fact, frontal and convective precipitations are characterized by large amounts of rain in the initial part of the event, such as BSC 1111 and 1110 - which together represent about $50 \%$ of the sample. Tropical-like cyclones are, instead, characterized by high amounts of rain in the middle part of the event, such as BSC 0010, 0110 and 0100 - which represent only about $8 \%$ of the sample.

Average values and standard deviations of the main parameters used to characterize SRP are listed in Table 4. The relationship of proportionality between the average value of $A^{+}$and of $A^{-}$is evidenced by their ratio; for BSC 1111 the ratio $\mu\left(A^{+}\right) / \mu\left(A^{-}\right)$assumes the value 155 while, for the teorically symmetrical BSC 0000 provides a much lower 4.7. This means that, on average, SRP having a BSC 0000 are closer to USRP than those characterized by a BSC 1111 .

With reference to the StAC, the lowest values correspond to the most advanced peaks - then to the BSC 1111, 1110, $\ldots$ - while the highest (lagged) to BSC 0000, 0100, ... These observations are not obvious to all BSC; e.g. BSC 0111 has, in average, a more advanced peak of BSC 1011 and 1101. It is therefore important to consider the information provided by SRP as a whole. 
Table 4. Sample size, average values $(\mu)$ and standard deviations $(\sigma)$ of $P_{\mathrm{ev}}, D_{\mathrm{ev}}, I_{30}, \mathrm{StAC}, A^{+}, A^{-}, A_{r}$ relevant to each BSC. In red, BSC with $\% N<2.5$; in blue, maximum values in each column as concerns BSC with $\% N \geq 2.5$.

\begin{tabular}{|c|c|c|c|c|c|c|c|c|c|c|c|c|c|c|c|}
\hline \multirow[b]{2}{*}{ BSC } & \multirow[b]{2}{*}{$N(-)$} & \multirow[b]{2}{*}{$\% N$} & \multicolumn{2}{|c|}{$P_{\mathrm{ev}}(\mathrm{mm})$} & \multicolumn{2}{|c|}{$D_{\mathrm{ev}}(\mathrm{h})$} & \multicolumn{2}{|c|}{$I_{30}\left(\mathrm{~mm} \mathrm{~h}^{-1}\right)$} & \multicolumn{2}{|c|}{ StAC (-) } & \multicolumn{2}{|c|}{$A^{+}(-)$} & \multicolumn{2}{|c|}{$A^{-}(-)$} & \multirow[b]{2}{*}{$A_{r}(-)$} \\
\hline & & & $\mu$ & $\sigma$ & $\mu$ & $\sigma$ & $\mu$ & $\sigma$ & $\mu$ & $\sigma$ & $\mu$ & $\sigma$ & $\mu$ & $\sigma$ & \\
\hline 0000 & 5652 & 12.4 & 23.2 & 25.7 & 883 & 699 & 12.7 & 11.1 & 0.757 & 0.199 & 0.045 & 0.052 & 0.210 & 0.070 & 0.272 \\
\hline 0001 & 929 & 2.0 & 25.3 & 25.9 & 818 & 658 & 16.9 & 14.9 & 0.666 & 0.116 & 0.191 & 0.028 & 0.150 & 0.037 & 0.173 \\
\hline 0010 & 2506 & 5.5 & 26.9 & 28.1 & 1077 & 846 & 9.3 & 7.8 & 0.562 & 0.239 & 0.092 & 0.068 & 0.090 & 0.034 & 0.192 \\
\hline 0011 & 4703 & 10.3 & 29.5 & 36.3 & 934 & 820 & 13.3 & 12.4 & 0.517 & 0.153 & 0.263 & 0.050 & 0.073 & 0.031 & 0.197 \\
\hline 0100 & 42 & 0.1 & 23.2 & 25.7 & 883 & 699 & 12.7 & 11.1 & 0.757 & 0.199 & 0.045 & 0.052 & 0.209 & 0.070 & 0.151 \\
\hline 0101 & 2 & 0.0 & & & & & & & & & & & & & \\
\hline 0110 & 1054 & 2.3 & 23.6 & 24.2 & 1061 & 718 & 9.4 & 6.9 & 0.450 & 0.269 & 0.156 & 0.078 & 0.043 & 0.022 & 0.201 \\
\hline 0111 & 3857 & 8.5 & 29.4 & 35.6 & 946 & 839 & 13.6 & 12.8 & 0.370 & 0.143 & 0.347 & 0.043 & 0.024 & 0.012 & 0.201 \\
\hline 1000 & 1704 & 3.7 & 18.0 & 18.9 & 836 & 622 & 9.9 & 7.7 & 0.660 & 0.339 & 0.049 & 0.051 & 0.139 & 0.051 & 0.239 \\
\hline 1001 & 228 & 0.5 & 26.2 & 30.8 & 932 & 772 & 12.4 & 11.5 & 0.553 & 0.243 & 0.188 & 0.128 & 0.098 & 0.076 & 0.239 \\
\hline 1010 & 963 & 2.1 & 23.2 & 25.6 & 1123 & 837 & 7.9 & 7.4 & 0.451 & 0.328 & 0.089 & 0.066 & 0.057 & 0.025 & 0.177 \\
\hline 1011 & 1343 & 2.9 & 23.3 & 23.9 & 990 & 716 & 9.7 & 7.7 & 0.437 & 0.255 & 0.260 & 0.048 & 0.036 & 0.019 & 0.183 \\
\hline 1100 & 344 & 0.8 & 15.9 & 12.5 & 707 & 503 & 9.5 & 6.3 & 0.528 & 0.394 & 0.069 & 0.051 & 0.091 & 0.029 & 0.184 \\
\hline 1101 & 33 & 0.1 & 18.5 & 14.9 & 767 & 539 & 11.8 & 8.3 & 0.495 & 0.328 & 0.203 & 0.027 & 0.046 & 0.008 & 0.121 \\
\hline 1110 & 4948 & 10.9 & 19.9 & 19.5 & 979 & 1151 & 8.8 & 6.8 & 0.318 & 0.336 & 0.178 & 0.080 & 0.023 & 0.022 & 0.283 \\
\hline 1111 & 17225 & 37.8 & 21.8 & 22.3 & 844 & 773 & 12.0 & 10.6 & 0.202 & 0.190 & 0.302 & 0.051 & 0.002 & 0.005 & 0.320 \\
\hline
\end{tabular}

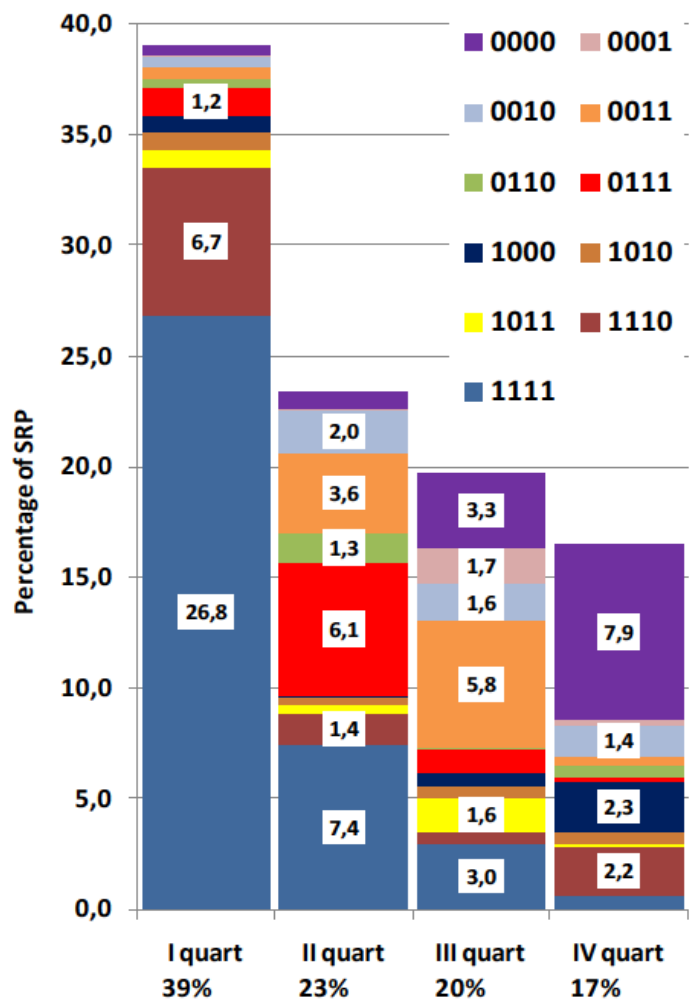

Fig. 4. Percentage of SRP associated with Huff's quartiles plotted according to their BSC; labels for percentages $<1 \%$ are not reported on the bars.
The relationship between SRP associated to Huff's quartiles and BSC is shown in Fig. 4. Almost all ErREv pertaining to the first quartile (about 85\%) fall in the BSC 1111 and 1110; ErREv pertaining to the 4th quartile are distributed in BSC 0000 (more than 46\%) but, in a significant proportion (about 13\%), also in BSC 1110 - which means that these Er$R E v$ (approximately one thousand $E r R E v$, pertaining to 4th quartile and BSC 1110) are characterized by a delayed peak, despite the amounts of accumulated rainfall in the other three quartiles are greater than those of USRP.

From the data in Table 4, BSC and average values of $D_{\mathrm{ev}}$ do not seem to be interrelated; rather, the average values of $I_{30}$ are dependent on the BSC. In particular, the four highest values of $I_{30}$ are characterized by BSC 0001, 0111, 0011 and 0000, which do not exceed the USRP in the first quarter of $D_{\mathrm{ev}}$. Finally, the two maximum averages of $P_{\mathrm{ev}}$ correspond to BSC 0011 and BSC 0111, and the two minimum averages to BSC 1110 and BSC 1000.

\section{Conclusions}

With respect to the rain events analyzed in previous studies, which are only a few hundred, those considered in this investigation are many thousands, and they are characterized by a very detailed time step. In particular, $45533 \mathrm{ErREV}$ events recorded at 155 Calabrian rain gauge stations between 1999 and 2008 were used to illustrate the proposed criterion for classifying the SRP. Following a statistical characterization of the database, the logical connections between the main SRP methods of analysis proposed in the literature (Huff, 
1967; Kottegoda and Kassim, 1991; Colosimo et al., 1996) were highlighted. Other elaborations allowed the differentiation of the profiles according to: (i) the season of occurrence, (ii) the range of duration of the rainfall event, (iii) the total rainfall of the event, and (iv) the maximum rainfall intensity in $30 \mathrm{~min}$.

The main conclusion that can be drawn from this work is that, in Calabria, the SRP curves which can be considered to define the design storm correspond only to 8 BSC out of 16 theoretically possible with frequencies higher than $2.5 \%$. Statistical analyses showed that SRP corresponding to BSC, which can be excluded, do not possess characteristics that make them interesting for hydrological applications (i.e., do not have, on average, high values of $\left.P_{\mathrm{ev}}, D_{\mathrm{ev}}, I_{30}\right)$.

A simple analysis of the number of SRP from the different BSC highlights:

1. the numerical preponderance of SRP with BSC 1111 and 0000 , respectively with 37.8 and $12.4 \%$ of all $\mathrm{Er}$ $R E v$;

2. almost $50 \%$ of the SRP has BSC 1111 or 1110 and low average values of StAC (i.e., most of the rain occurs with high intensity during the initial part of the event).

Accordingly, the criterion for classification of rain profiles proposed in this study improved the general knowledge framework - for instance, by excluding some storm structures characterized by very low sampling frequency.

Other interesting considerations can be summarized as follows:

- notable differences were found for Calabrian $E r R E v$, with respect to the analysis carried out according to Huff (1967). In Calabria, the average $D_{\mathrm{ev}}$ of ErREv have decreasing values going from the 1 st to the 4 th quartile; corresponding standard deviations show increasing values, but the differences between such values are minor, contrary to what was reported for Illinois, where a general association was found between $D_{\mathrm{ev}}$ and quartile (Huff, 1967).

- The SRP set was characterized in terms of storm structure, highlighting their variability on the base of various physical characteristics of the storms.

- Quantitative assessments of some physical features $\left(A^{+}, A^{-}\right.$, StAC,$\left.A_{r}\right)$ were presented about the structure of the SRP, helping to improve the input to hydrological models. In particular, the following features were considered: (i) the sampling frequency of BSC, and (ii) the BSC functional links with $D_{\mathrm{ev}}, P_{\mathrm{ev}}, I_{30}, \mathrm{StAC}, A^{+}$and $A^{-}$.

The classification criterion of SRP proposed here, thanks to the considerable sample size, (i) allows the specification of the ErREv characteristics by improving the application of
Huff's approach, (ii) enhances usability as an input for mathematical models and (iii) integrates with other important contributions in literature.

Consequently, after selecting the quartile with the highest sampling frequency, the curve for the 50\% quantile was used.

This study provides a more accurate characterization of the structure and of physical characteristics of rain events in Calabria and also provides the necessary elements for applications targeted at specific needs. For example, when considering the large number of small Calabrian watersheds in which flash floods are very frequent, the SRP taken as input for a rainfall-runoff model might be characterized by a BSC 1111, which has the largest sampling frequency, a high $I_{30}$, a high $P_{\mathrm{ev}}$ and a low StAC average. The quantiles 10 to $90 \%$ could be used to assess the possibility that SRP, different but statistically equivalent, may give rise to conditions of different severity in terms of flood peaks. However, if interest were directed to the runoff volume (and not the peak discharge), the SRP could be adopted corresponding to the BSC 0011 and 0111 , which on average possess the maximum $P_{\mathrm{ev}}$ values.

Further developments of this study may include a joint analysis of the most interesting combinations of hydrological situations: for example, the SRP having, at the same time, $P_{\mathrm{ev}}$ and $\mathrm{I}_{30}$ exceeding pre-fixed values, or those having, yet at the same time, $I_{30}$ and $D_{\text {ev }}$ exceeding pre-fixed values, etc. In addition, supplementary improvements may result from a more advanced probabilistic analysis and interpretation of SRP using mathematical functions. Finally, a more stringent analysis must be conducted closer to the spatial aspects related to propagation of SRP in space, according to orography, to the weather peculiarities of rainfall events, and to the characteristics taken along the path by their distinctive parameters.

Acknowledgements. The authors are very grateful to the referees and to the editor for the fruitful suggestions that allowed us to improve the paper. They also thank Stefano Luigi Gariano for the effective management of the rainfall database.

Edited by: A. Mugnai

Reviewed by: two anonymous referees

\section{References}

ARPACal-Regione Calabria http://195.216.128.112:81/banca_dati/ midmar/banca_dati.php, access: 22 June 2009.

Bellecci, C., Federico, S., Casella, G., Avolio, E., Lo Feudo, T., and Sisca, M.: Intense Precipitation in southern Italy, in: New Trends in Hydrology, Editoriale BIOS, Cosenza, Italy, 57-74, 2002.

Chuckwuma, G. O. and Schwab, G. O.: Procedure for developing design hyetographs for small watersheds, Transactions ASAE, 26(5), 1386-1389, 1983.

Colosimo, C., Mendicino, G., and Terranova, O.: An analysis of rainfall profiles of an experimental basin (Turbolo creek basin-Italy). In Proceedings of the Conferencia Internacional de 
Geologia Ambiental y Ordenacion del territorio, Granada, Spain, 22-25 April 1996, 173-186, 1996.

Colosimo, C. and Copertino, V. A.: Analisi dei profili di pioggia in un piccolo bacino urbano, in: Proceedings of seminar on "Deflussi Urbani”, Cosenza, Italy, 25-32, 1984.

Huff, F. A.: Time distribution of heavy rain storms in Illinois, Water Resour. Res., 3 (4), 1007-1019, 1967.

Kottegoda, N. T. and Kassim, A. H. M.: Classification of storm profiles using crossing properties, J. Hydrol., 127(1/4), 37-53, 1991.

Natural Environment Research Council (NERC): Flood Studies Report, Vol. 2, Meteorological studies, Whitefriars Press Ltd., London, 81 pp., 1975.
Terranova, O.: Caratteristiche degli eventi pluviometrici a scala giornaliera in Calabria, In: Proceedings of XXIX Convegno di Idraulica e Costruzioni Idrauliche, Trento, Italy, 7-10 September 2004, 343-350, 2004.

Vukmirovic, V. D. and Despotovic, J. Z.: Statistical methods of storm Analysis, in: Proceedings of a Specialized Seminar on Rainfall - The Basis for Urban Runoff Design and Analysis, Copenhagen, Denmark, 24-26 August, 85-92, 1983.

Wischmeier, W. H. and Smith, D. D.: Predicting rainfall erosion losses - a guide to conservation planning, Agricultural Handbook 537, USDA, Agricultural Research Service, Washington, DC, 58 pp., 1978. 